\title{
Crystallization Behaviors of Polypropylene/Polyamide-6 Blends Modified by a Maleated Thermoplastic Elastomer
}

\author{
Hongzhi LiU, ${ }^{1,4}$ Tingxiu XIE, ${ }^{3}$ Ying Zhang, ${ }^{2,4}$ Yuchun Ou, ${ }^{1, \dagger}$ and Guisheng YANG ${ }^{1,3, \dagger}$ \\ ${ }^{1}$ Key Laboratory of Engineering Plastics, Joint Laboratory of Polymer Science \& Materials, \\ Institute of Chemistry, Chinese Academy of Sciences, Beijing 100080, People's Republic of China \\ ${ }^{2}$ State Key Laboratory of Polymer Physics \& Chemistry, Joint Laboratory of Polymer Science \& Material, \\ Institute of Chemistry, Chinese Academy of Sciences, Beijing 100080, People's Republic of China \\ ${ }^{3}$ Shanghai Genius Advanced Materials Co., Ltd., Shanghai, 201109, People's Republic of China \\ ${ }^{4}$ Graduate School of the Chinese Academy of Sciences, Beijing, People's Republic of China
}

(Received February 10, 2005; Accepted August 1, 2005; Published January 15, 2006)

\begin{abstract}
In this paper, with the maleated thermoplastic elastomer (TPEg) as a compatibilizer of polypropylene (PP)/polyamide-6 (PA6) blends, the effects of interfacial compatibilization on the crystallization behaviors of the blends were investigated by differential scanning calorimetry (DSC). Compared with the binary blend, the addition of TPEg significantly affected the crystallization behaviors of the individual component in the blends, especially those of PA6. The presence of TPEg weakened the nucleating role of the PA6 on the PP matrix. The increasing concentration of the compatibilizer caused a decrease in crystallization temperature $\left(T_{\mathrm{c}}\right)$ and crystallization enthalpy $\left(\Delta H_{\mathrm{c}}\right)$ associated with PA6. When TPEg amount was added up to $24 \mathrm{wt} \%$, the crystallization of PA6 at its bulk $T_{\mathrm{c}}$ was almost completely suppressed and the complete concurrent crystallization of PA6 and PP matrix took place. In the case of PP/PA6/TPEg blend containing $24 \mathrm{wt} \% \mathrm{TPEg}$, it was found that the disappearance of bulk PA6 crystallization peak was independent of the cooling rate and annealing time, but can be introduced by self-nucleation experiments. The above fractionated crystallization phenomenon was attributed to the reduction in PA6 particle size due to compatibilization role of added TPEg, which leads to the lack of the active heterogeneities. The crystalline morphologies under isothermal conditions were also observed by polarized optical microscopy (POM). [DOI 10.1295/polymj.38.21]

KEY WORDS Polypropylene (PP) / Polyamide-6 / Crystallization / Compatibilization / Morphology /
\end{abstract}

Compatibilization strongly affects the blend phase morphology and as such, it may also influence the crystallization behaviors of the blend. Both morphology and crystallization behaviors are closely related with the final properties of the blend, a profound understanding of the crystallization behavior and the resulting semicrystalline structure in polymer blends is necessary for effective manipulation and control of their properties from both scientific and industrial point of view. However, the general influence of a compatibilizer on the crystallization behavior of an immiscible polymer blend system is still far from being well understood, especially for crystalline polymer/crystalline polymer. ${ }^{1}$

In a given crystalline/crystalline blends containing the compatibilizer, the crystallization behaviors of the two blends components are generally affected by the following factors: the size of dispersion, ${ }^{2-5}$ the type of compatibilizer ${ }^{2,3,6-8}$ and its degree of miscibility with and one or both of the blend components, the amount of compatibilizer added, ${ }^{9,10}$ the amount of interface created, ${ }^{10}$ and other effects (e.g. the mixing ratio of both crystalline components ${ }^{2,6}$ and mixing procedure $^{11}$ ).

At present, many studies have been carried out on the crystallization behavior of various compatibilized polypropylene/polyamide-6 (PP/PA6) blend systems. It has been demonstrated that addition of the compatibilizers, such as PP-g-MA ${ }^{2,9,12-14}$ or SEBS-g-MA, ${ }^{2,10}$ significantly affected the crystallization behaviors of blend component. In our previous work, a maleated thermoplastic elastomer (TPEg) was successfully used as an interfacial modifier of PP/PA6 uncompatibilzed blends ${ }^{15}$ and their dynamic rheological properties and microstructures were investigated. ${ }^{16}$

As a continuation of the previous work, this paper focused on the effects of interfacial compatibilization on crystallization behaviors of the individual components in PP/PA6 blends with differential scanning calorimetry (DSC) and the resulting development of morphologies was also presented in terms of polarized optical microscopy (POM) and transmission electron microscopy (TEM). 


\section{EXPERIMENTAL}

\section{Materials}

The PP used in this study was a commercial product (PP2401) from Yanshan Petro. \& Chem. Co., with melt flow index $(\mathrm{MFI})=3.95 \mathrm{~g} / 10 \mathrm{~min}\left(230^{\circ} \mathrm{C}\right.$, $2.16 \mathrm{~kg}$ ). PA-6 was supplied by Shanghai Plastics Production Factory No. 18 (Shanghai, China) with the relative viscosity equal to 2.6-2.9. The interfacial modifier (TPEg) was a maleic anhydride grafted mixture of poly(octene-ethylene) (POE) rubber and a semicrystalline polyolefin plastic (60/40 by weight). Details about the functionalization procedure were reported elsewhere. ${ }^{17}$ Its grafting maleic anhydride content was about $1 \mathrm{wt} \%$. The POE is a commercial product from Dupont DOW Elastomers (Wilmington, DE) under the trade name Engage 8445. Its octene content and MFI were $9.5 \%$ and $3.5 \mathrm{~g} / 10 \mathrm{~min}$, respectively. The blends were prepared using a $\Phi 30$ twinscrew extruder with an L/D ratio of 23.2 (SHJ-30, Nanjing Plastic Machinery Co., Ltd., Nanjing, China) at $250 \mathrm{rpm}$. The barrel temperature was $220^{\circ} \mathrm{C}$. The weight percentage of PA-6 was fixed at $30 \mathrm{wt} \%$ in all the blends. The blends were pelletized, dried, and injection-molded into standard tensile, flexural, and Izod impact test specimens with an injection-molding machine (SZ-160/80 NB, Ningbo Plastics Machinery Co., Ltd., Ningbo, Zhejiang Province, China) at 220$230^{\circ} \mathrm{C}$.

\section{Differential Scanning Calorimetry (DSC)}

Thermal analysis was performed with Perkin-Elmer Diamond DSC under nitrogen atmosphere using a cooling and heating rate of $10^{\circ} \mathrm{C} / \mathrm{min}$. About $4 \mathrm{mg}$ samples obtained by cutting from the center of injection-molded specimens were used. All the samples were firstly heated to $250^{\circ} \mathrm{C}$ and maintained at that temperature for $5 \mathrm{~min}$ to erase previous thermal history. The crystallization and melting thermograms were taken from the subsequent cooling and second heating cycles. The nonisothermal crystallization temperature $\left(T_{\mathrm{c}}\right)$ and melting temperature $\left(T_{\mathrm{m}}\right)$ values were determined from the crystallization and melting peak values, respectively. All enthalpy values were corrected from the blend composition.

In order to confirm the origin behind fractionated crystallization, the following self-nucleation procedure $^{18}$ is applied in the investigation: erasing thermal history and creating a initial standard state. The samples were first heated to $250^{\circ} \mathrm{C}$ and kept there for $5 \mathrm{~min}$. Subsequently, the samples were cooled to room temperature at a cooling rate of $10^{\circ} \mathrm{C} / \mathrm{min}$. Heating to self-nucleation temperatures $\left(T_{\mathrm{S}}\right)$ at a heating rate of $10^{\circ} \mathrm{C} / \mathrm{min}$ and kept there for $5 \mathrm{~min}$, followed by the
DSC cooling scan from $T_{\mathrm{s}}$ at the same cooling rate. The corresponding cooling curves were recorded.

\section{Particle Size Analysis}

To clearly visualize the dispersed PA6 particles, the surfaces of cryogenically fractured specimens were firstly etched in formic acid (88\%) solution for $2 \mathrm{~h}$ at $50{ }^{\circ} \mathrm{C}$ to remove PA6 domains and then vigorously washed with anhydrous ethanol. After drying, the etched fracture surfaces were sputtered with gold prior to SEM observation (Hitachi S-1300F). In order to determine the PA6 particle size, at least 600 pits left after etching for each blend composition were analyzed using Phoenix EDS image analyzer (EDAX, USA). The volume average diameter $\left(d_{v}\right)$ were calculated from the following relationships:

$$
d_{v}=\frac{\sum n_{i} d_{i}^{4}}{\sum n_{i} d_{i}^{3}}
$$

where $n_{i}$ is the number of particles having the true particle diameter $d_{i}$.

\section{Transmission Electron Microscopy (TEM)}

Specimens were prepared by cryomicrotoming of the injection-molded strands at $-80{ }^{\circ} \mathrm{C}$ using a diamond knife. The cutting direction was perpendicular to the molded strands. Afterwards, the specimens were exposed to the vapor of ruthenium tetraoxide $\left(\mathrm{RuO}_{4}\right)$ for $20 \mathrm{~min}$ to develop contrast between different components prior to TEM observation (Hitachi H-800).

\section{Polarized Optical Microscopy (POM)}

Polarized optical microscopy measurements equipped with Panasonic GP-230 CCD system were made on a LTS (Link Scientific Instruments Ltd.) hot-stage and Olympus BH-2 Optical Microscope. Samples in the form of flat chips were cut from injection-molded specimens with $20-30 \mu \mathrm{m}$ in thickness using Leica RM-2155 microtome (Germany). Samples were first heated to $250^{\circ} \mathrm{C}$ under a nitrogen atmosphere, held for $5 \mathrm{~min}$ to eliminate all residual nuclei, and firstly cooled quickly to $160^{\circ} \mathrm{C}$ for isothermal crystallization. After some time, the samples were again quenched $130^{\circ} \mathrm{C}$ to crystallize isothermally. The corresponding micrographs were taken at appropriate intervals.

\section{RESULTS AND DISCUSSIONS}

\section{Differential Scanning Calorimetry (DSC)}

The crystallization thermograms for the noncompatibilized PP/PA6 blend (70/30, by weight) after as well as for both unextruded virgin homopolymers are shown in Figure 1. The separate crystallization exotherms of the individual components in the noncom- 


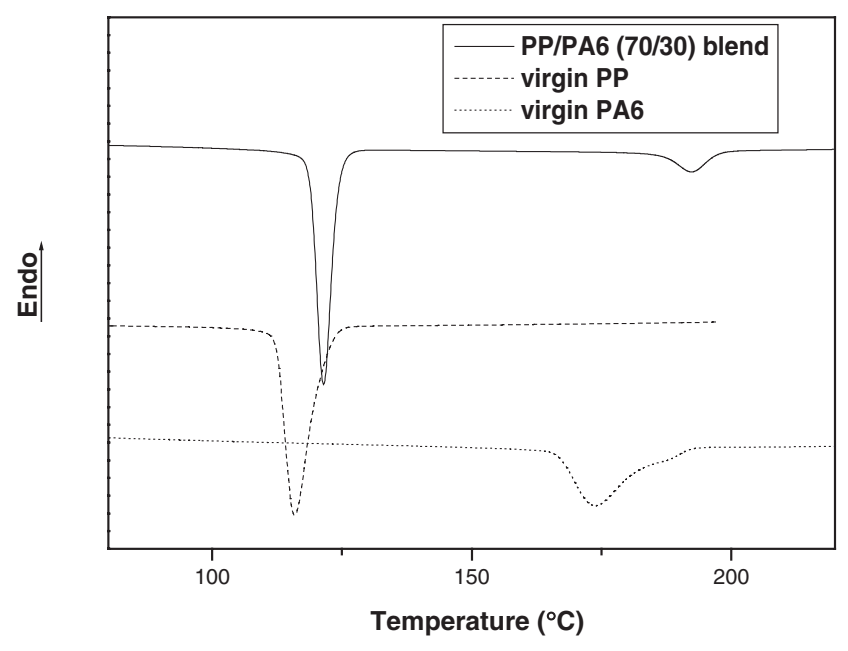

Figure 1. DSC cooling thermograms of virgin PP, virgin PA6 and the noncompatibilized PP/PA6 (70/30, by weight) blend.

patibilized blend can be clearly distinguished, typical of the immiscible blend. The crystallization temperature $\left(T_{\mathrm{c}}\right)$ is $116.9^{\circ} \mathrm{C}$ for virgin $\mathrm{PP}$ and $173.8^{\circ} \mathrm{C}$ with a shoulder on the high temperature side at about $187^{\circ} \mathrm{C}$ for virgin PA6. The $T_{\mathrm{c}}$ rises to 120.2 and $192.4^{\circ} \mathrm{C}$ for PP and PA6, respectively, in noncompatibilized blend. The above shift in the $T_{\mathrm{c}}$ of the PP matrix to a higher temperature has been ascribed to the nucleating effect of the crystallized PA6 component in the blend, as described elsewhere. ${ }^{13,14,19}$

It is interesting that PA6 component in the noncompatibilized PP/PA6 blend exhibits a higher crystallization temperature (above $18.6^{\circ} \mathrm{C}$ or so, compared to the virgin PA6). This results is quite similar to the behavior on the PA6 homopolymer found by Khanna et $a .^{20,21}$ and has been explained by a more ordered molecular arrangement that persists in the molten state due to the stabilizing effect of hydrogen bonding after melt extrusion of PA6. The virgin PA6 is thought to be disordered and to stay disordered in the molten state, again due to hydrogen bonding. During processing, shearing is supposed to likely induce locally ordered regions, which are presumed to act as nucleating sites, causing an increase in the crystallization rate. ${ }^{21}$ Moreover, such orientation memory of PA6 was retained even at $280^{\circ} \mathrm{C}$ for $5 \mathrm{~min}$ (much above the equilibrium melting temperature), while non-polar polymers were not influenced in terms of crystallization behavior by the processing history unlike polar polymers. In our case, in order to further make clear the role of PP matrix in the increase, $T_{\mathrm{c}}$ values of PA6 component in the extruded PP/PA6 (70/30) pellets $\left(191.8^{\circ} \mathrm{C}\right)$ and its subsequent injection-molded sample $\left(192.4^{\circ} \mathrm{C}\right)$ are compared, in addition to the extruded PA6 pellets $\left(190.3^{\circ} \mathrm{C}\right)$ and its subsequent injection-molded sample $\left(190.5^{\circ} \mathrm{C}\right)$. It is found that these $T_{\mathrm{c}}$ value are almost the same. Therefore, the above

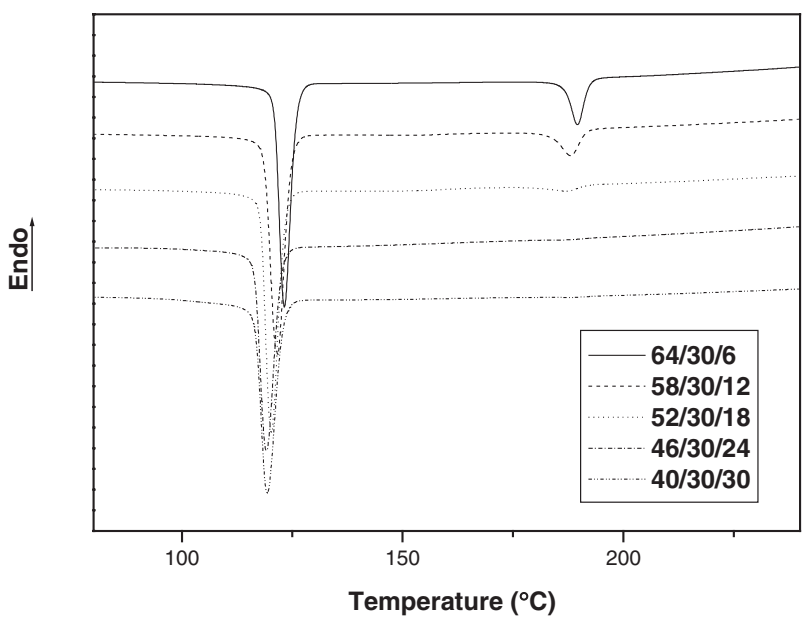

Figure 2. DSC cooling thermograms of PP/PA6/TPEg blends.

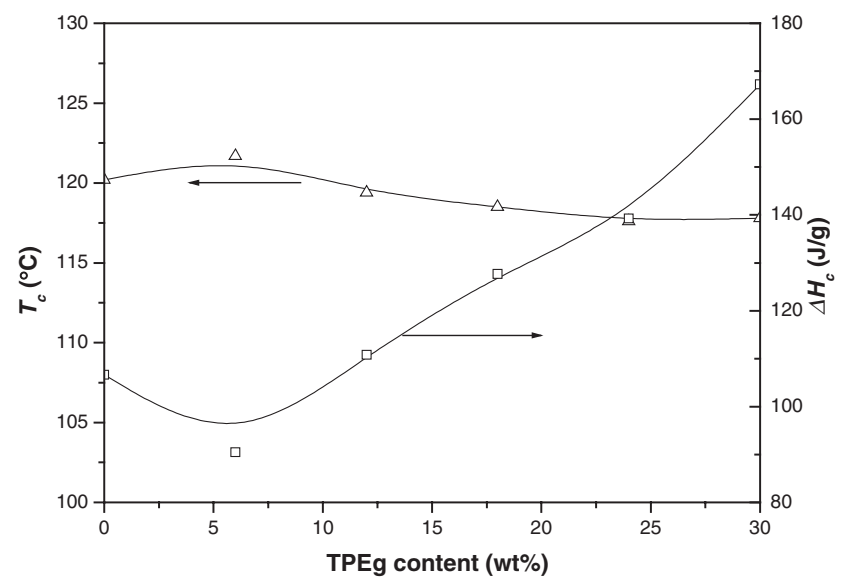

Figure 3. Dependence of crystallization enthalpy $\left(\Delta H_{\mathrm{c}}\right)$ and crystallization temperature $\left(T_{\mathrm{c}}\right)$ for PP component on TPEg concentration, respectively.

increase in $T_{\mathrm{c}}$ of PA6 in the PP/PA6 binary blend should result from the initial twin-screw extruding step, in which molecular chain extension and orientation are increased under role of melt shearing. Secondly, the presence of PP matrix does not seem to account for the aforementioned remarkable increase in $T_{\mathrm{c}}$ values of PA6 component.

Figure 2 shows the crystallization thermograms for the PP/PA6 blends compatibilized with various concentration of TPEg. The dependence of $T_{\mathrm{c}}$ and normalized $\Delta H_{\mathrm{c}}$ of PP component upon TPEg content for PP/ PA6/TPEg blends is presented in Figure 3. It can be observed that at the low TPEg content (6 wt \%), the $T_{\mathrm{c}}$ of PP component in the compatibilized blend is even somewhat higher than in the uncompatibilized one. However, the further increase in the concentration of TPEg causes a progressive decrease. Since the $T_{\mathrm{c}}$ of PP matrix in the PP/TPEg $(75 / 25)$ binary blend is almost same as that of virgin $\mathrm{PP}\left(116.7^{\circ} \mathrm{C} v s .116 .9^{\circ} \mathrm{C}\right)$, the above drop in $T_{\mathrm{c}}$ value is attributed to the inhibition 


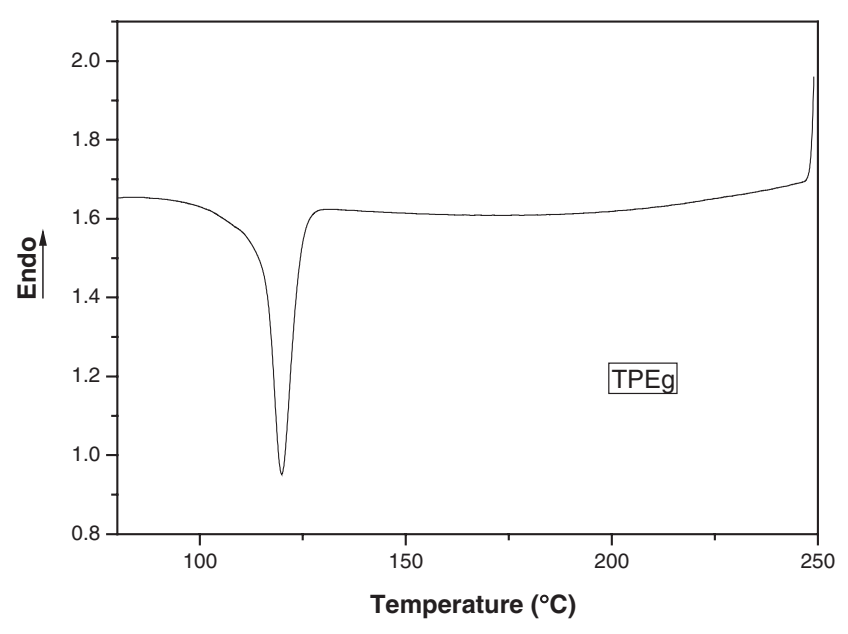

Figure 4. DSC cooling thermograms of TPEg.

of the above PA6 nucleation role by encapsulating interlayer around PA6 particles formed during reactive compatibilization. This interlayer has been confirmed by the previous $\mathrm{RuO}_{4}$-stained TEM micrographs. ${ }^{16}$ Based on the above result, it can also be concluded that encapsulation for dispersed PA6 particles become complete progressively with TPEg concentration.

Addition of the compatibilizer initially results in a slight decrease of $\Delta H_{\mathrm{c}}$ value of PP component (6 wt \% TPEg), followed by a marked increase with the TPEg content. The minimum value of $\Delta H_{\mathrm{c}}$ may be due to the decrease in the aforementioned nucleating effect of PA6 on the PP matrix. When the TPEg content increases further, the resulting decrease in size of PA6 particles causes a portion of finer PA6 to coincidently crystallize with PP matrix (i.e. fractionated crystallization), which contributes to the increase in $\Delta H_{\mathrm{c}}$ value of PP component. Besides, the semicrystalline polyolefin component in the TPEg may also make a certain contribution to the above increase in the above $\Delta H_{\mathrm{c}}$ value when added TPEg amount is higher. Figure 4 presents the crystallization thermograms for pure TPEg. It can be observed that an exothermic peak occurs in $120^{\circ} \mathrm{C}$, very close to the $T_{\mathrm{c}}$ of PP component in PP/PA6/TPEg blends.

The addition of the compatibilizer to the PP/PA6 blends significantly changes the magnitude of crystallization peak of PA6 as well as its peak position. Table I lists the values $T_{\mathrm{c}}$ and $\Delta H_{\mathrm{c}}$ for PA6 component in the PP/PA6/TPEg blends with a fixed PA6 content. With increasing the TPEg concentration up to $18 \mathrm{wt} \%$, the value of $T_{\mathrm{c}}$ and $\Delta H_{\mathrm{c}}$ for PA6 in the blends shows a slow decrease, accompanied by a broadening of the crystallization peak, as seen in Figure 2. But the value of $T_{\mathrm{c}}$ for PA6 in the compatibilized blends is still remarkably higher than that for virgin PA6. This result suggests that the above extrusion processing effects, i.e. melt shearing, still exist in the PP/PA6 blends with
Table I. Crystallization temperature $\left(T_{\mathrm{c}}\right)$ and crystallization enthalpy $\left(\Delta H_{\mathrm{c}}\right)$ for PA6 component in PP/PA6 $6^{\mathrm{a}}$ blends compatibilized with different amount of TPEg

\begin{tabular}{lrrrrrr}
\hline & \multicolumn{7}{c}{ TPEg content (wt \%) } \\
\cline { 2 - 7 } & \multicolumn{1}{c}{0} & \multicolumn{1}{c}{6} & \multicolumn{1}{c}{12} & \multicolumn{1}{c}{18} & $24^{\mathrm{b}}$ & $30^{\mathrm{b}}$ \\
\hline$T_{\mathrm{c}}\left({ }^{\circ} \mathrm{C}\right)$ & 192.4 & 189.5 & 188.1 & 187.2 & - & - \\
$\Delta H_{\mathrm{c}}(\mathrm{J} / \mathrm{g})$ & 46.7 & 47.6 & 36.0 & 12.3 & - & - \\
\hline
\end{tabular}

${ }^{\text {a }}$ PA6 content fixed at $30 \mathrm{wt} \%$; ${ }^{\mathrm{b}}$ no detectable crystallization peak for PA6 at usual position.

the compatibilizer. However, when the content of TPEg in the blends is 24 and $30 \mathrm{wt} \%$, the normal crystallization peak attributable to PA6 disappears until the bulk crystallization peak for PP begins to occur. This crystallization behaviors of PA6 dispersed phase could be explained in terms of a fractionated crystallization process, which is mainly caused by the reduction of the particle size. The larger droplets that contain the heterogeneities usually active at low undercoolings in the bulk polymer will crystallize at identical temperatures to that polymer in the bulk. In the smaller droplets the character of nucleation changes: these droplets contain other types of less efficient heterogeneities and will nucleate at the undercooling necessary for those heterogeneities to become active. Finally, the smallest droplets that do not contain any heterogeneities will only nucleate at the largest undercooling, since a greater undercooling is usually needed in order to generate homogeneous nuclei. ${ }^{22-24}$ Hence, during cooling from melt, crystallization of the minor component may take place in several steps with widely different undercoolings. The series of crystallization exotherms observed in the DSC curves can be interpreted as a crystallization of different groups of droplets occurring at specific and independent undercooling. Of course, the crystallization of the minor component can also take place coincidently with the major blend component, namely concurrent crystallization.

The effect of TPEg content on PA6 dispersed phase size for PP/PA6 blends is presented in Figure 5, characteristic of the emulsion curves. ${ }^{25}$ The addition of TPEg causes a rapid decrease in the size up to a critical concentration of $18 \mathrm{wt} \%$. Above this critical concentration $\left(C_{\text {crit }}\right)$, which represents the saturation of the interface by the modifier, the particle size attains a steady-state value $\left(d_{\text {equil }}\right) .{ }^{26}$ An overall decrease of about a fifth in $d_{v}$ value is observed. Such significant reduction is attributed to a decrease of the interfacial tension and the coalescence inhibition of the dispersed PA6 particles due to the formation of TPE-g-PA6 interfacial copolymer. ${ }^{27}$

When only $6 \mathrm{wt} \%$ TPEg is added, the size of PA6 particles is significantly reduced with respect to the noncompatibilized blend due to the compatibilization 


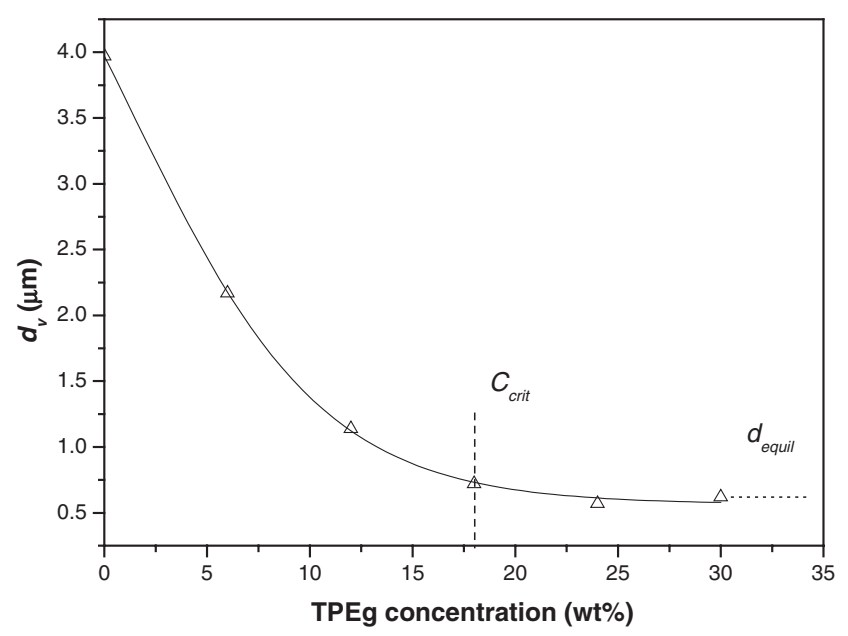

Figure 5. Dependence of volume average diameter $\left(d_{v}\right)$ of PA6 particles on TPEg concentration in PP/PA6/TPEg blends.

role of TPEg. Due to the fixed PA6 content in the blends (30 wt \%), the number of PA6 particles becomes more. In this case, however, the size of PA6 particles may be still larger than the critical size, below which the fraction crystallization takes place. Hence the crystallization of PA6 in the blend still remains at its bulk. It can be observed from Table I that the $\Delta H_{\mathrm{c}}$ values of PA6 are almost the same for PP/ PA6 (70/30) and PP/PA6/TPEg (64/30/6) blends. With the further increasing concentration of TPEg, the average particle size of PA6 further decreases. This causes a portion of the smaller PA6 particles below the critical size to crystallize at the temperature lower than the usual bulk $T_{\mathrm{c}}$ for PA6. With TPEg content up to $24 \mathrm{wt} \%$, almost all PA completely crystallize coincidently with PP matrix. Moon et al. ${ }^{9}$ reported that in the PP/PA6 (70/30) blend, the addition of only $1.5 \mathrm{phr}$ PP-g-MA resulted in the occurrence of almost completely concurrent crystallization of PA6 with PP matrix. Ohlsson et al. ${ }^{10}$ observed that at the SEBS-g-MA concentration of $10 \mathrm{wt} \%$ and higher, the PA6 crystallization peak for PP/PA6/SEBS-g-MA blends appeared as a low temperature shoulder, or a peak overlapping the normal crystallization peak of PP matrix. The difference may be mainly due to the different degree of interfacial compatibilization associated with different compatibilizers.

Because the crystallization behaviors of polymers usually exhibit remarkable dependence on time or cooling rate, the cooling curves of the PP/PA6/TPEg blend with $24 \mathrm{wt} \%$ TPEg at various cooling rates are presented in Figure 6. Whatever the cooling rate is, only one crystallization peak occurs between $110^{\circ} \mathrm{C}$ and $130^{\circ} \mathrm{C}$. No bulk exothermic peak of PA6 can be observed, still characteristic of the fractionated crystallization. It suggests that no dependence on cooling rate exists for the disappearance of PA6 bulk crystalliza-

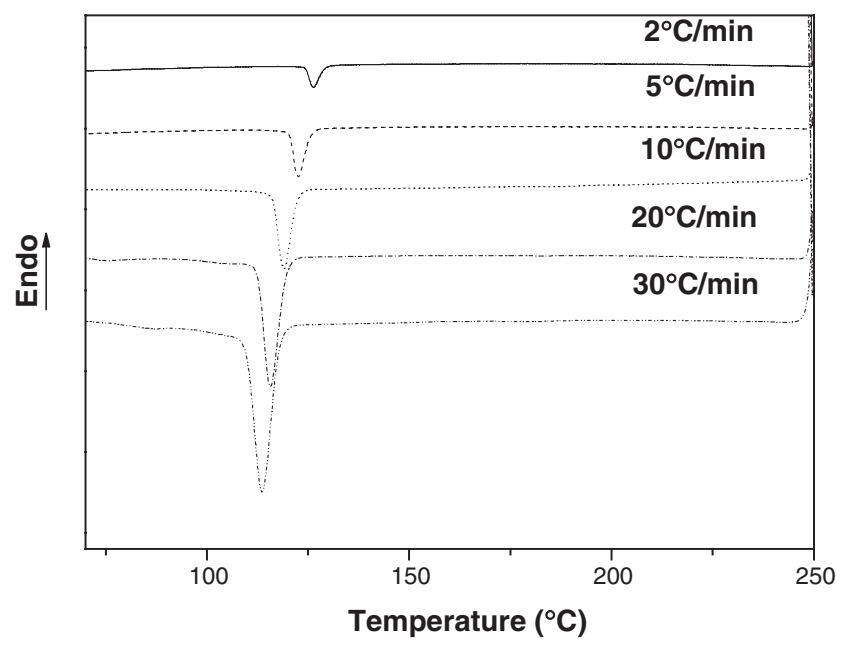

Figure 6. The cooling curves of PP/PA6/TPEg containing $24 \mathrm{wt} \%$ TPEg at various cooling rates.

tion peak. In addition, as is expected, the peak temperature shifts to a lower temperature region with the increase of the cooling rates. This observation is a common phenomenon for semicrystalline polymer crystallized nonisothermally. When polymer was undergoing crystallization at lower cooling rate, it had relatively long time lying within the temperature range that promoted sufficient mobility of segments for the growth of crystallites; but when cooled down at a relatively rapid rate, segments were frozen before the formation of regular crystallite, thereby decreasing the crystallization temperature. Furthermore, the annealing experiment at $160^{\circ} \mathrm{C}$ was also performed with the $24 \mathrm{wt} \%$ TPEg blend. At this temperature, it is usually believed that only PA6 crystallization can take place. After erasing thermal history at $250^{\circ} \mathrm{C}$, the blend sample was quenched to $160^{\circ} \mathrm{C}$ at $200^{\circ} \mathrm{C} / \mathrm{min}$ and then annealing for different time, respectively. Then, the subsequent heating run was performed from 160 to $250^{\circ} \mathrm{C}$ to check whether the crystallization of PA6 during the annealing occurred (Figure 7). It is found that no distinguished exothermic peak can be observed in the three heating curves, which implies that no crystallization of PA6 occurs during the annealing. In order to further confirm the origin behind fractionated crystallization, self-nucleation experiments are also performed. With this method the nucleation density is increased enormously by heating up the material within the self-nucleation regimes where small crystal fragments are still present in the melt. These fragments will act as predetermined nuclei for recrystallization upon cooling and can cause the nucleation process to start at higher temperatures than would normally be the case. Figure 8 gives the cooling curves after the self-nucleation at $222^{\circ} \mathrm{C}$. The bulk crystallization of PA6 component recurs in the cooling thermograms. Therefore, the disappearance of PA6 bulk crys- 


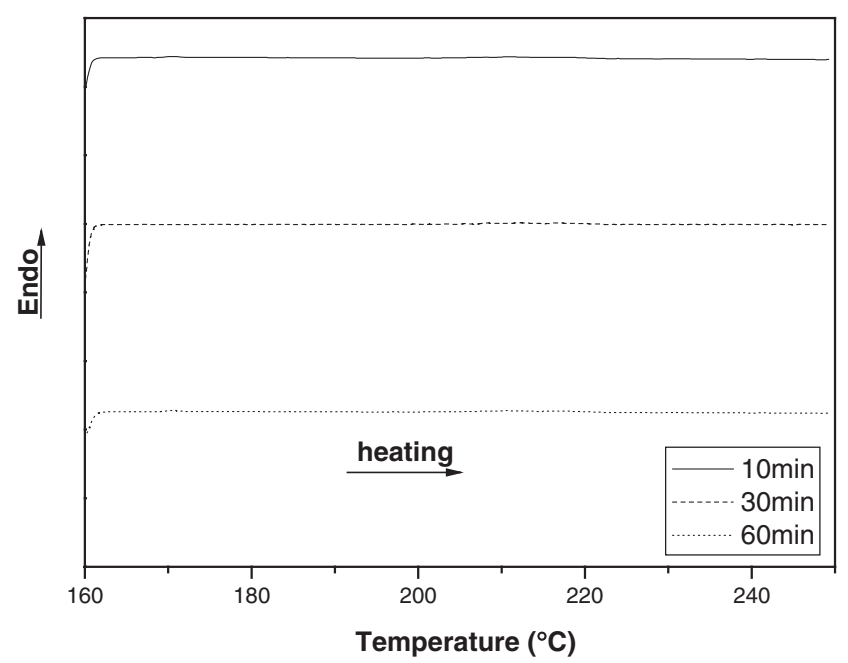

Figure 7. The subsequent heating curves of PP/PA6/TPEg containing $24 \mathrm{wt} \% \mathrm{TPEg}$ after annealing at $160^{\circ} \mathrm{C}$ for three different times.

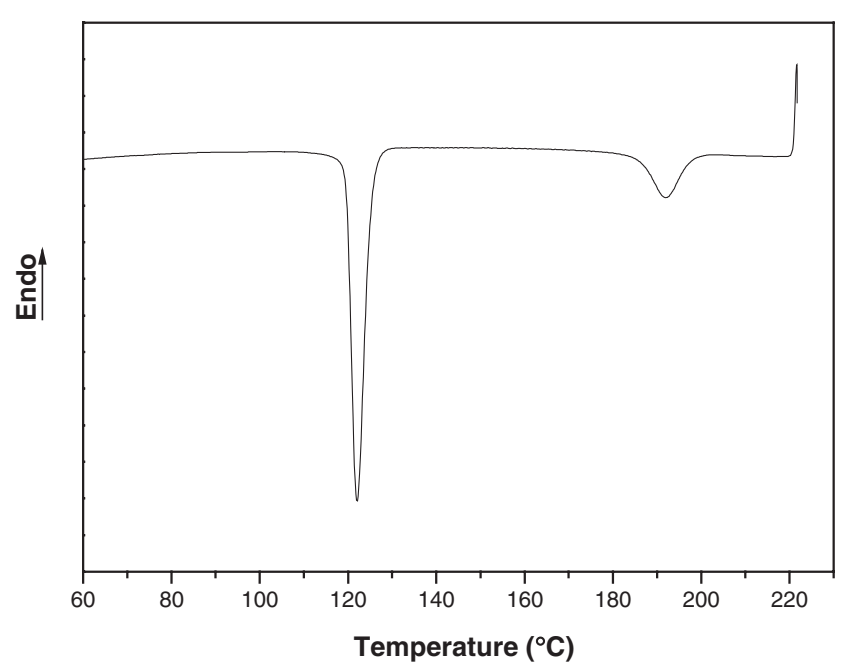

Figure 8. The cooling curves of PP/PA6/TPEg containing $24 \mathrm{wt} \%$ TPEg after self-nucleation at $222{ }^{\circ} \mathrm{C}$ for $5 \mathrm{~min}$.

tallization peak is as a result of the lack of active heterogeneities under the used undercooling.

DSC second heating traces of PP/PA6 blends with various amount of TPEg are shown in Figure 9. In all the blends, one can observe both PP and PA6 components melting peaks occurring at their usual positions corresponding to the two bulk polymers, which means that PP and PA6 do not co-crystallize but retarded crystallization or coincident crystallization with PP matrix takes place for PA6 component in the blends, as mentioned above. With increasing the content of the compatibilizer, the melting temperature $\left(T_{\mathrm{m}}\right)$ of PP almost remained unchanged, whereas some noticeable changes occurring during the melting region of PA6 are observed. As shown in Figure 9, during the second heating period, the PA6 exhibits a bimodal melting peak with a main peak at approximately

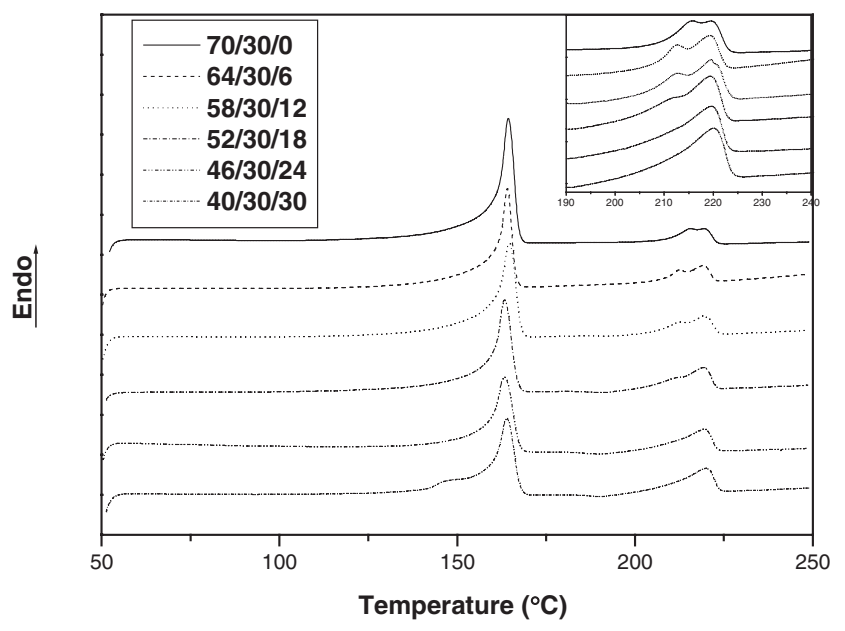

Figure 9. DSC heating thermograms of PP/PA6 modified with different content of TPEg. Inset: The magnification of PA6 melting region in the main graph.

$219^{\circ} \mathrm{C}$ and a lower temperature shoulder at approximately $212{ }^{\circ} \mathrm{C}$. With addition of TPEg, the intensity of the shoulder peak gradually becomes weak and seems to disappear in the case of $24 \mathrm{wt} \%$ TPEg, whereas the position of high melting peak remains unchanged. Wilkinson et al. ${ }^{28}$ also found the same phenomenon in the PP/PA6/SEBS-g-MA ternary blends and attributed the shoulder peak to the secondary crystallization of PA6 component. ${ }^{29-31}$ The extruded PA6 used in the study also exhibits this bimodal melting behavior under the same measurement condition. The conditions that reduce the mobility of chain segments after primary crystallization has occurred, such as rapid cooling ${ }^{28}$ and crystallization at high degrees of undercooling, ${ }^{2,28}$ can suppress the development of the low temperature shoulder and produce a melting endotherm with a single peak.

\section{Polarized Optical Microscopy (POM)}

In order to further investigate crystalline morphologies of blends, POM observation under isothermal condition is also performed. Because the prolonged time changes the crystallization morphology very slightly, $40 \mathrm{~min}$ for $160^{\circ} \mathrm{C}$ and $30 \mathrm{~min}$ for $130^{\circ} \mathrm{C}$ are selected, respectively. Figure 10 shows the crystallization morphologies of PP/PA6/TPEg blends under crossed polarizers at $160^{\circ} \mathrm{C}$. At this temperature, only PA6 component in the blends can usually crystallize. It can be observed that as the content of TPEg increases, the domain size of PA6 decrease, and the amount of PA6 that crystallizes at this temperature decreases. No perceptible crystal is observed at $160^{\circ} \mathrm{C}$ when content of TPEg reaches $24 \mathrm{wt} \%$, which agrees with the DSC results in Figure 7. Based on the above DSC discussion, this can be attributed to the occurrence of fraction crystallization of PA6 components. 

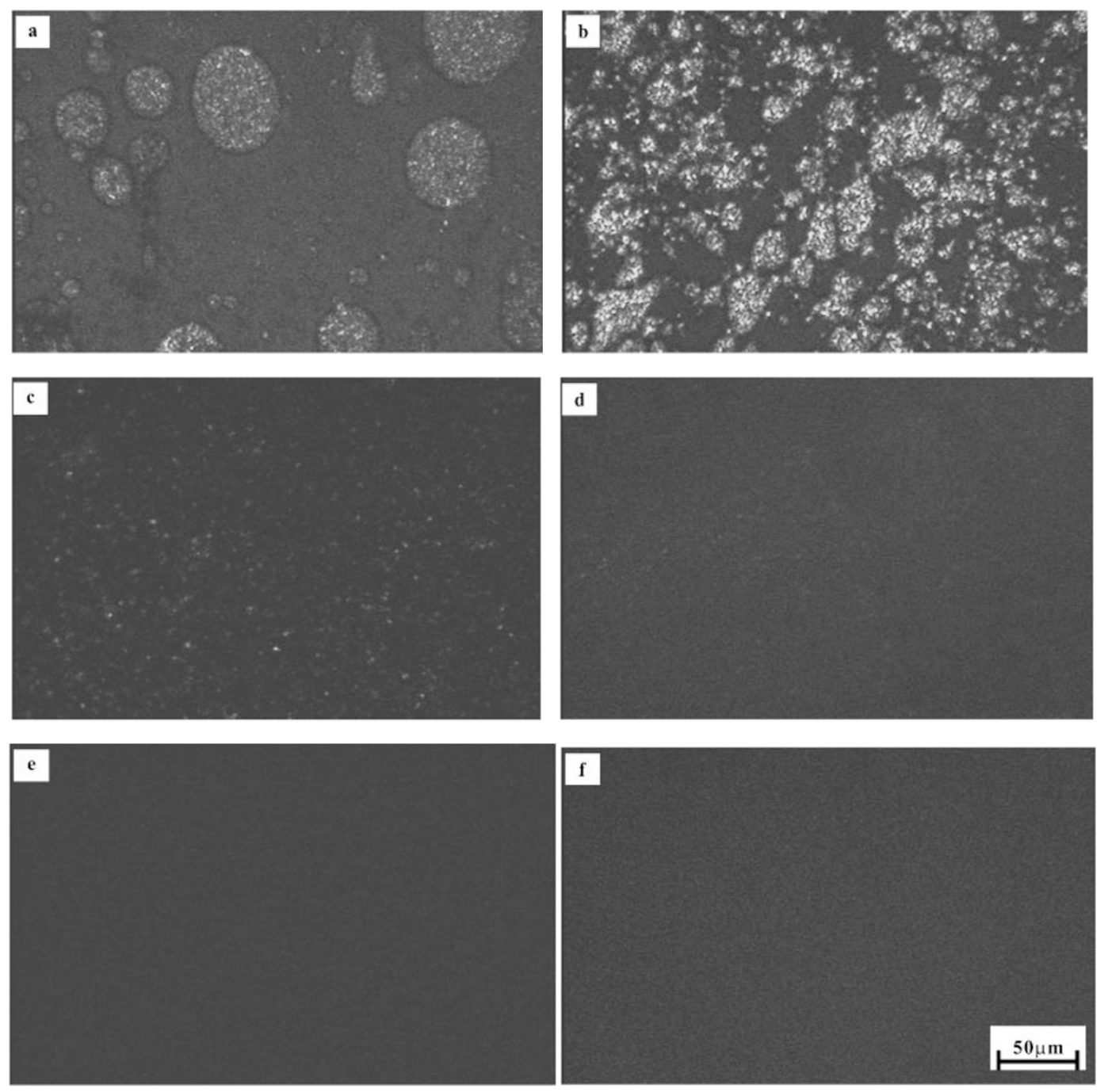

Figure 10. POM micrographs of the isothermal crystallization of PP/PA6/TPEg blends taken at $160{ }^{\circ} \mathrm{C}$ for $40 \mathrm{~min}$ : (a) $70 / 30 / 0$; (b) $64 / 30 / 6$; (c) $58 / 30 / 12$; (d) $52 / 30 / 18$; (e) $46 / 30 / 24$; (f) $40 / 30 / 30$.

Figure 11 shows the POM micrographs of the blends isothermally crystallized at $130^{\circ} \mathrm{C}$, at which both PP and PA6 may crystallize. When no TPEg or only $6 \mathrm{wt} \%$ one is added (Figures 11a and 11b), one can observe that crystals of both PP and PA6 are well phase-separated and the boundary between them may be easily distinguished. Again, the evolution of crystallization morphology during the isothermal crystallization process at $130^{\circ} \mathrm{C}$ clearly reveals that the crystallization of PP preferentially nucleates at the interface of previously crystallized PA6 for the above both blends (not shown here). Obviously, the crystallized PA6 particles act as nucleating agents or heterogeneities in the crystallization of PP. On the other hand, with an identical isothermal crystallization treatment in the presence of higher TPEg concentration, the PP and PA6 crystals phases seem to become more mixed. And it is very difficult to distinguish a segregated PA6 phase from PP one. In this case, the morphological evolution during the isothermal crystalliza- tion reveals as well that nucleating effect of PA6 in the crystallization of PP is difficult to be observed. Very distorted and incomplete spherulites with smaller size are formed since the more homogeneously dispersed PA6 seriously disturbs the interlaminar aggregation of PP spherulites. The observation is similar to that in the PP/PA6 (70/30) blends with PP-g-MA as a compatibilizer. ${ }^{13}$ This may be ascribed to the more homogeneous dispersion of PA6 due to an improved interfacial interaction. They can be reflected by a significant increase in the viscosity with increasing TPEg content, as described previously. ${ }^{16}$

However, it is noticeable that when further increasing TPEg content up to $30 \mathrm{wt} \%$, the size and amount of the crystal become larger again. This phenomenon may be ascribed to the occurrence of agglomeration of PA6 particles, for which concurrent crystallization with PP matrix takes place. This assumption can be confirmed in the following $\mathrm{RuO}_{4}$-stained TEM micrographs of PP/PA6/TPEg blends, as shown in 

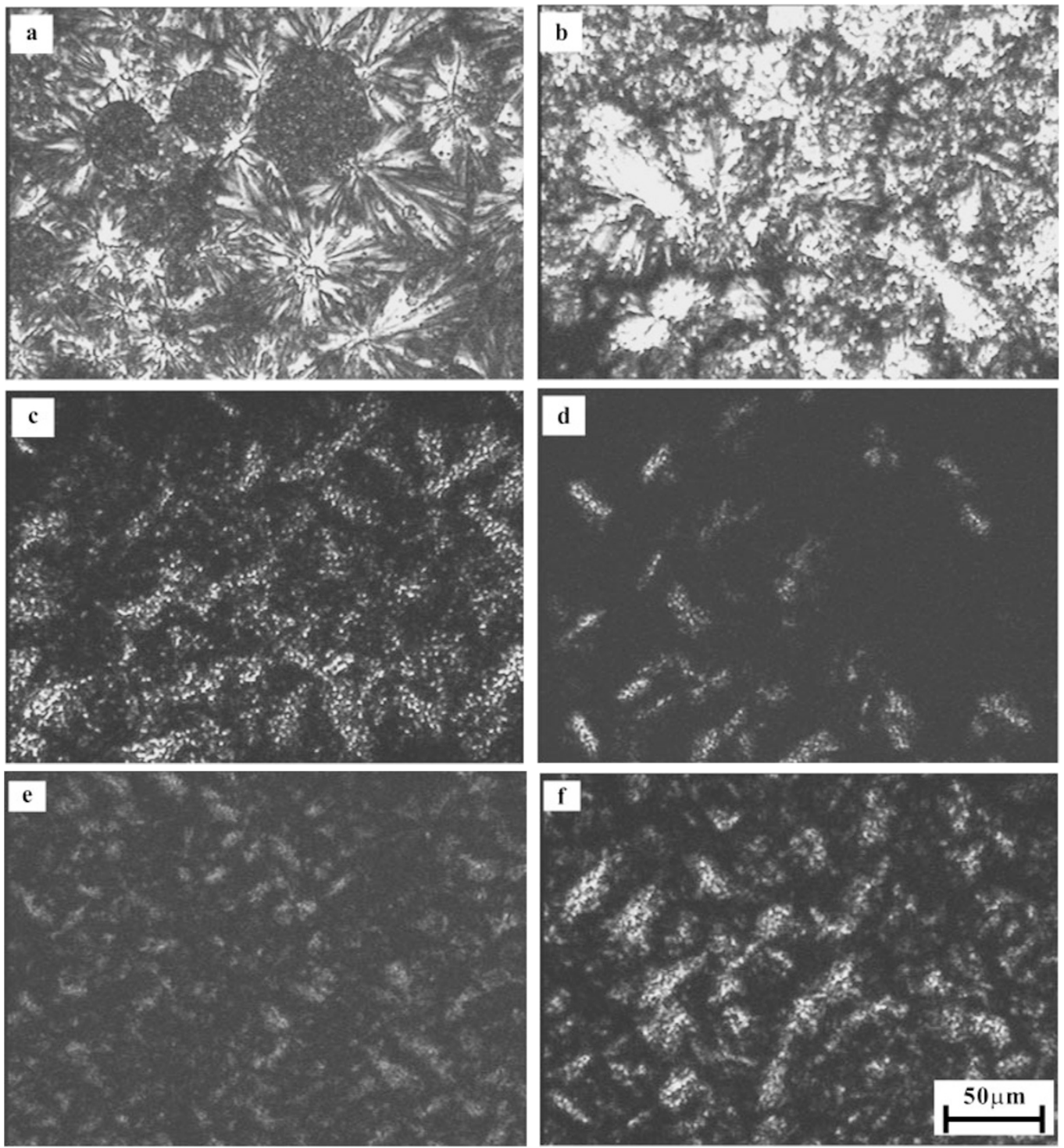

Figure 11. POM micrographs of the isothermal crystallization of PP/PA6/TPEg blends taken at $130{ }^{\circ} \mathrm{C}$ for $30 \mathrm{~min}:(\mathrm{a}) 70 / 30 / 0$; (b) $64 / 30 / 6$; (c) $58 / 30 / 12$; (d) $52 / 30 / 18$; (e) $46 / 30 / 24$; (f) $40 / 30 / 30$.

Figure 12. At lower TPEg concentration, the particles are discretely dispersed in the matrix. With addition of TPEg concentration up to $24 \mathrm{wt} \%$, some agglomeration seems to occur in the form of cluster. At the higher TPEg concentration, the degree of agglomeration becomes more obvious.

\section{CONCLUSIONS}

Because of the interfacial compatibilization, the PP/PA6/TPEg blends exhibited more complex crystallization behaviors, compared to corresponding uncompatibilized blend. In the compatibilized blends, the addition of TPEg diminished the nucleating role of the PA6 component on the PP bulk with increasing TPEg content. This result implies the enhancement of encapsulation degree for PA6 particles.

The crystallization temperature $\left(T_{\mathrm{c}}\right)$ of PA6 compo- nent in the blends leveled off down as the concentration of TPEg increased. The further addition of TPEg caused a progressive reduction in the magnitude of the crystallization peak at the usual $T_{\mathrm{c}}$ for PA6, which suggested a decrease in crystallization enthalpy $\left(\Delta H_{\mathrm{c}}\right)$. When TPEg concentration reached $24 \mathrm{wt} \%$, the crystallization peak of PA6 at its usual $T_{\mathrm{c}}$ disappeared, while the normalized crystallization enthalpy of PP in the blend increased markedly with TPEg concentration. These above results implied the occurrence of fractionated crystallization for PA6 component, which attributed to the significant reduction in PA6 particles size because of the interfacial compatibilization. As for the compatibilized blend with $24 \mathrm{wt} \% \mathrm{TPEg}$, it was found that the disappearance of bulk PA6 crystallization peak was independent of the cooling rate and annealing time. Its self-nucleation experiments further confirmed that the origin of fractionated crystalliza- 

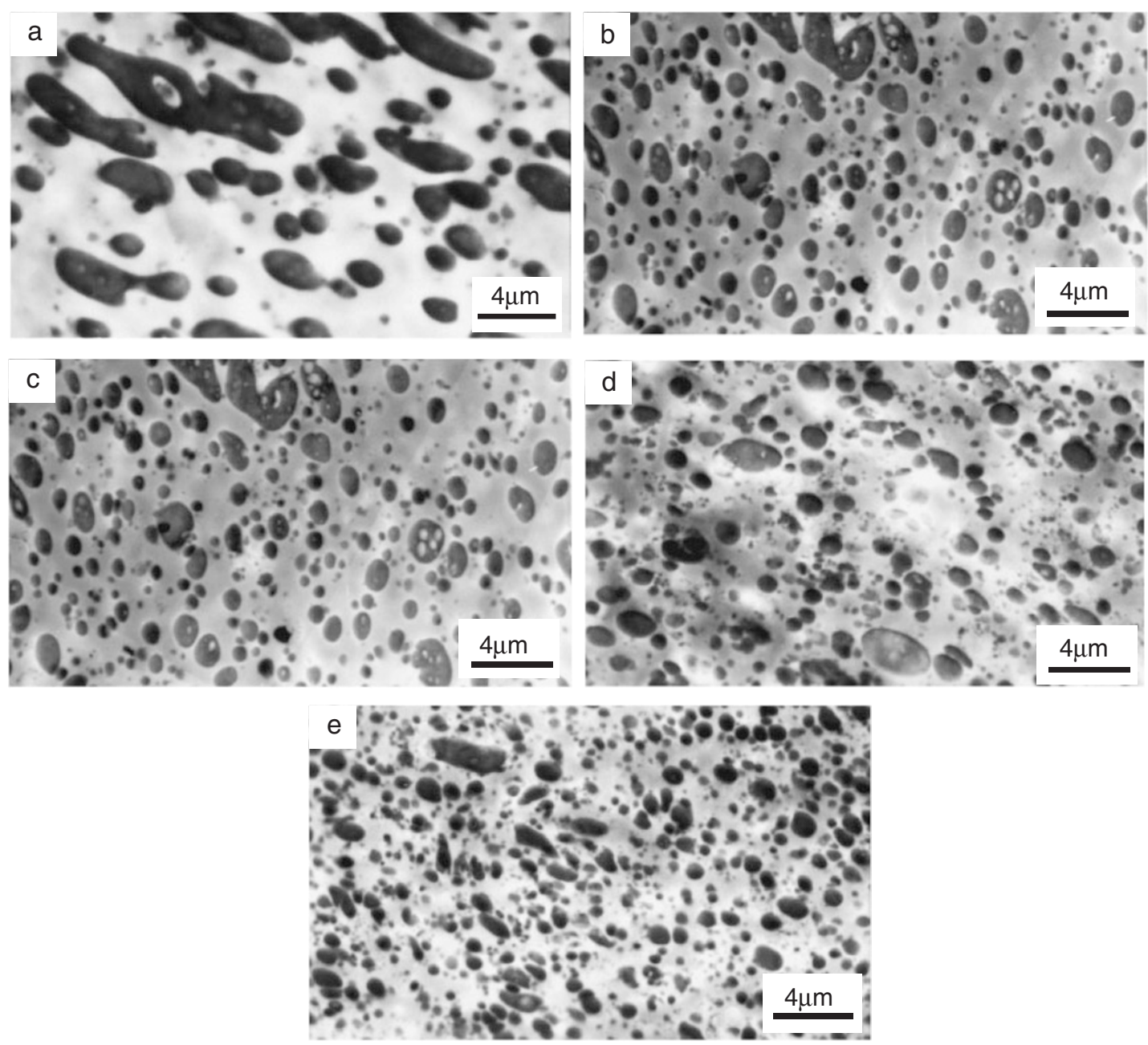

Figure 12. $\mathrm{RuO}_{4}$-stained TEM micrographs of PP/PA6/TPEg blends: (a) 64/30/6; (b) 58/30/12; (c) 52/30/18; (d) 46/30/24; (e) $40 / 30 / 30$.

tion is the lack of active heterogeneities under the undercooling used.

The POM observation under isothermal condition demonstrated that addition of TPEg dramatically changed the crystalline morphologies of PP/PA6 blends. However, it needed to be noticed that when TPEg content increased from 24 to $30 \mathrm{wt} \%$, the size and amount of observable crystals under POM became larger. The phenomenon might result from the occurrence of agglomeration of PA6 domain, which was also approved by TEM observation.

Acknowledgment. The authors are grateful to both National Natural Science Key Foundation of China (Contract No. 10032010) and National Natural Science Foundation of China (Contract No. 10272005) for financial support.

\section{REFERENCES}

1. G. Groeninckx, M. Vanneste, and V. Everaert, in "Polymer Blends Handbooks,” L. A. Utracki, Ed., Kluwer Academic,
Netherlands, Oordrecht, 2003, chapt. 7, p 280.

2. O. T. Ikkala, R. M. Holsti-Miettinen, and J. Seppala, J. Appl. Polym. Sci., 49, 1165 (1993).

3. V. Flaris, A. Wasiak, and W. Wenig, J. Mater. Sci., 28, 1685 (1993).

4. T. Tang and B. Huang, J. Appl. Polym. Sci., 53, 355 (1994).

5. R. Holsti-Miettinen, M. Geino, and J. Seppala, J. Appl. Polym. Sci. 57, 573 (1995).

6. R. Holsti-Miettinen and J. Seppala, Polym. Eng. Sci., 32, 868 (1992).

7. U. Plawky and W. Wenig, J. Mater. Sci. Lett., 13, 863 (1994).

8. S. M. B. Nachtigall, A. H. O. Flexis, and R. S. Mauler, J. Appl. Polym. Sci., 88, 2492 (2003).

9. Y.-S. Moon, B.-K. Ryoo, and J.-K. Park, J. Polym. Sci., Part B: Polym. Phys., 32, 1427 (1994).

10. B. Ohlsson, H. Hassander, and B. Tornell, Polymer, 39, 6705 (1998).

11. B. Ohlsson, H. Hassander, and B. Tornell, Polymer, 39, 4715 (1998).

12. D. Shi, J. Yin, Z. Ke, Y. Guo, and R. K. Li, J. Appl. Polym. Sci., 91, 3742 (2004).

13. C. Marco, G. Ellis, M. A. Gomez, J. G. Fatou, and J. M. Arribas, I. Compoy, and A. Fontecha, J. Appl. Polym. Sci., 
65, 2665 (1997).

14. I. Compoy, J. M. Arribas, M. A. M. Zaporta, M. A. Gomez, and J. G. Fatou, Eur. Polym. J., 31, 475 (1995).

15. Y.-C. Ou, Y.-G. Lei, X.-P. Fang, and G.-S. Yang, J. Appl. Polym. Sci., 91, 1806 (2004).

16. H.-Z. Liu, T.-X. Xie, Y.-C. Ou, X.-P. Fang, and G.-S. Yang, Polym. J., 36, 754 (2004).

17. Z.-Z. Yu, Y.-C. Ou, Z.-N. Qi, and G.-H. Hu, J. Polym. Sci., Part B: Polym. Phys., 36, 1987 (1998).

18. D. J. Blundel, A. Keller, and A. J. Kovacs, J. Polym. Sci., Part B: Polym. Lett., 4, 481 (1966).

19. S. H. Jafari and A. K. Gupta, J. Appl. Polym. Sci., 71, 1153 (1997).

20. Y. P. Khanna, A. C. Reimschuessel, A. Banerjie, and C. Altman, Polym. Eng. Sci., 28, 1600 (1988).

21. Y. P. Khanna, R. Kumar, and A. C. Reimschuessel, Polym. Eng. Sci., 28, 1607 (1988).

22. H. Frensch, P. Harnischfeger, and B.-J. Jungnickel, in "Multiphase Polymer: Blends and Ionomer,” L. A. Utracki and
R. A. Weiss, Ed., American Chemical Society, Washington, DC, 1989, ACS Symp. Ser., 395, p 101.

23. H. Frensch and B.-J. Jungnickel, Colloid Polym. Sci., 267, 16 (1989).

24. H. Frensch and B.-J. Jungnickel, Plast., Rubber Compos. Process. Appl., 16, 5 (1991).

25. B. D. Favis, Polymer, 35, 1552 (1994).

26. J.-C. Lepers, B. D. Favis, and C. Lacroix, J. Polym. Sci., Part B: Polym. Phys., 37, 939 (1999).

27. J.-C. Lepers, B. D. Favis, and R. J. Tabar, J. Polym. Sci., Part B: Polym. Phys., 35, 2271 (1997).

28. A. N. Wilkinson, M. L. Clemens, and V. M. Harding, Polymer, 45, 5239 (2004).

29. W.-Y. Chiang, W.-D. Yang, and B. Pukanszky, Polym. Eng. Sci., 32, 641 (1992).

30. B. S. Hsiao, K. H. Gardner, D. Q. Wu, and B. Chu, Polymer, 34, 3996 (1993).

31. J. Kim, M. E. Nichols, and R. E. Robertson, J. Polym. Sci., Part B: Polym. Phys., 32, 887 (1994). 